\title{
Standpunkt
}

\section{Didaktik zwischen Dogmatik und Problemfokussierung}

\author{
Wolfgang Stegmaier"
}

\begin{abstract}
Die Staatsrechtslehrertagung im Oktober 2019 stand ganz im Zeichen des Verhältnisses von Öffentlichem Recht und Privatrecht. In Vorträgen wurde unter anderem die kategoriale Unterscheidung von Öffentlichem Recht und Privatrecht infrage gestellt, und es wurden die Verschränkungen öffentlich-rechtlicher und privatrechtlicher Regime im Verwaltungsrecht fokussiert und diskutiert. ${ }^{1}$ Zuvor hatte sich, bereits im September 2019, auch die Zivilrechtslehrertagung mit Themen an der Schnittstelle zwischen Öffentlichem Recht und Privatrecht beschäftigt. ${ }^{2}$ Beide Veranstaltungen haben - wieder einmal - gezeigt, wie durchlässig die erst mit dem Aufblühen der Pandektenwissenschaft im 19. Jahrhundert scharf gezogene Grenzlinie zwischen Öffentlichem Recht und Privatrecht ist. Kaum eine rechtliche Problemstellung ist in der Praxis vorstellbar, in der nicht sowohl Gesichtspunkte des Öffentlichen Rechts als auch privatrechtliche Aspekte mit einspielen würden. Vor diesem Hintergrund stellt sich die Frage, ob die strenge Unterscheidung in der juristischen Ausbildung zwischen beiden Rechtsgebieten weiterhin sinnvoll ist oder ob den Studenten der Zugang durch stärker thematisch ausgerichtete Lehrformate erleichtert werden könnte.
\end{abstract}

Die dogmatische Unterscheidung zwischen Privatrecht und Öffentlichem Recht geht bekanntlich auf den römischen Rechtsgelehrten Ulpian (170-228 n. Chr.) zurück und bildet seitdem eine der Grundlinien des Gemeinen Rechts und unserer heutigen Rechtsordnung. Bis zum 19. Jahrhundert hatte diese Zweiteilung indessen nicht die absolute Bedeutung, die ihr seitdem beigemessen wird. Carl Gottlieb Svarez (1746-1798), der Verfasser des Preußischen Allgemeinen Landrechts von 1794, legte diese Unterscheidung beispielsweise zwar seinen juristischen Vorlesungen vor dem preußischen Kronprinzen (1791-1792), dem späteren König Friedrich Wilhelm III., zugrunde, ${ }^{3}$ untergliederte das Landrecht selbst hingegen in der im 18. Jahrhundert noch selbstverständlichen Anordnung der Rechtsmaterien in zwei Teile, einem „Sachenrecht“ und einem „Personenrecht“; 4 in beiden Teilen finden sich neben Bestimmungen des Privatrechts auch solche des Öffentlichen Rechts. Diese

* Prof. Dr. Wolfgang Stegmaier ist Inhaber einer Professur für Immobilienrecht an der Fachhochschule Westküste in Heide (Holstein).

1 Tagungsprogramm unter: https://www.staatsrechtslehrertagung.de/programm.html, abgerufen am 21. Juli 2020, 15:00 Uhr.

2 Tagungsprogramm unter: http://www.zlv-info.de/fileadmin/PDF/Tagung/Referenten_und_Themen_d er_Zivilrechtslehrertagungen_2019.pdf, abgerufen am 21. Juli 2020, 15:05 Uhr.

3 Vergleiche dazu: Krause, Carl Gottlieb Svarez. Die Kronprinzenvorlesungen 1791/1792. StuttgartBad Cannstatt, 2000.

4 Wobei beide Begriffe einen anderen, wesentlich weitreichenderen Inhalt hatten als im heutigen juristischen Sprachgebrauch. 
Vorgehensweise entsprach der aufklärerischen Intention der Zeit: Die Gesetze sollten „in einer zusammenhängenden Ordnung [...] und dergestalt allgemein verständlich vorgetragen werde[n], daß ein jeder Einwohner des Staats, dessen natürliche Fähigkeiten durch Erziehung nur einigermaßen ausgebildet sind, die Gesetze, [...] selbst lesen, verstehen, und in vorkommenden Fällen sich nach den Vorschriften derselben gehörig achten könne. “5 Die Verständlichkeit, die intellektuelle $\mathrm{Zu}$ gänglichkeit standen also ganz im Vordergrund, Privatrecht und Staatsrecht waren zweckmäßig, aber bunt gemischt.

Diese in höchstem Maße pragmatische Zielsetzung didaktischer Erschließung des Rechts darf man zwar grundsätzlich auch der Arbeit der beiden BGB-Kommissionen unterstellen. Die Rechtslehre im 19. Jahrhundert war indessen von der Pandektenwissenschaft dominiert, und die Juristenausbildung fokussierte sich erst wieder mit der Einführung des Bürgerlichen Gesetzbuchs auf positiviertes Recht, ohne allerdings öffentlich-rechtliche und privatrechtliche Materien $\mathrm{zu}$ verbinden, obschon sie doch in der Praxis in den seltensten Fällen berührungsfrei bleiben; menschliches Handeln, das nach privatrechtlichen Regeln zu beurteilen ist, tangiert für gewöhnlich auch öffentlich-rechtliche Bestimmungen, und wenn es sich dabei lediglich - etwa im Handelsrecht - um Steuerrecht oder - etwa im Deliktsrecht um Vorschriften des Ordnungswidrigkeitenrechts handeln sollte.

$\mathrm{Ob}$ deshalb jedoch auch die didaktische Ausrichtung in der juristischen Ausbildung geändert werden sollte, darf bezweifelt werden. Jura ist traditionell ein Verständnisfach, das in seiner vollen Breite nur erfassbar ist, wenn die Grundstrukturen gelehrt werden, auf denen die Rechtsordnung aufbaut. Dies ist die Voraussetzung, um sich dem Recht wissenschaftlich zu nähern, um Rechtswissenschaft zu betreiben. Einzelwissen ist daran anknüpfbar, Wissen kommt so zu Wissen. Nicht ein zahlreiches Einzelwissen ist es, welches für die Grundlagen rechtswissenschaftlicher Ausbildung die Ankerpunkte für die Anknüpfung neuen Wissens liefert, sondern das Verständnis für Systeme. Die Funktionsweise des Privatrechts ist jedoch grundlegend verschieden von der Funktionsweise des Öffentlichen Rechts. Dem Privatrecht liegt ein - zwei- oder mehrpolares - Ausgleichsbedürfnis zu Grunde: Interessenskonflikte zwischen Individuen, die sich aus teils gleichlaufenden, teils entgegengesetzten Bedürfnissen ergeben, sollen mithilfe des Privatrechts einem geregelten Ausgleich zugeführt werden; Privatrecht ist damit nichts anderes als die Friedensordnung unter Einzelnen, ${ }^{6}$ gerade bei unbestimmten und wertausfüllungsbedürftigen Rechtsbegriffen wird dies deutlich: Die im $\mathbb{1 3 8}$ BGB bemühten „guten Sitten" sind nichts anderes als die Frage, was im Verhältnis zwischen Personen noch als erträglich angesehen wird, eine Leistungserbringung nach „Treu und Glauben“ gemäß $\$ 242$ BGB erfolgt nur, wenn Gläubiger- und Schuldnerinteresse

5 Publikationspatent zum Allgemeinen Gesetzbuch für die preußischen Staaten vom 20. März 1791, zitiert nach: Klein, Annalen der Gesetzgebung und Rechtsgelehrsamkeit in den Preussischen Staaten. 8. Band. Berlin und Stettin, 1791.

6 Vergleiche dazu: Stegmaier, Das Preußische Allgemeine Landrecht und seine staatsrechtlichen Normen, Berlin, 2014, Seite 22; Stegmaier, NJW 2020, 1642, 1644 f. 
angemessen berücksichtigt und ausgeglichen sind, und sämtliche Klauselbeispiele des $\ 308$ BGB - um ein letztes Beispiel zu nennen - hängen von einer Wertung ab, bei der das Interesse des Kunden sowie des Verwenders einer Allgemeinen Geschäftsbedingung ausgeglichen werden soll.

Ganz anders verfährt der Rechtsanwender in der öffentlich-rechtlichen Arbeit. Nicht der Interessensausgleich unter Einzelnen ist hier die dominierende Determinante, sondern die teils tradierten, teils dem Verfassungsrecht entnommenen Grundsätze der Verwaltungstätigkeit, die auf Erfüllung des Staatszwecks gerichtet sind, und zwar im Sinne eines Staates als „Wirklichkeit der sittlichen Idee“, der mehr ist als die Addition der Individualwillen: nämlich eines Staates, der durch Selbsterhaltung nach außen und Befriedung nach innen die Möglichkeit des Wohls eines jeden Bürgers schafft und sichert und daher auch Interessen eigener Art formulieren muss. ${ }^{7}$

Diese ganz unterschiedlichen Ansatzpunkte werden beispielsweise virulent, wenn es um die Maßstäbe der Ausübung von Ermessen geht. „Billiges Ermessen“, etwa im $\$ 315$ BGB und dem römischen Recht nachgebildet, setzt aequitas ${ }^{8}$ voraus und ist unter Berücksichtigung der Interessen beider Parteien und des in vergleichbaren Fällen Üblichen festzustellen, ${ }^{9}$ orientiert sich also am Interesse der Beteiligten, wohingegen „pflichtmäßiges Ermessen“ im Verwaltungsrecht unter anderem dem Grundsatz der Verhältnismäßigkeit genügen muss und daher - insoweit einseitig daran gemessen wird, ob nicht eine für den Betroffenen weniger beeinträchtigende Maßnahme zum gleichen Ziele führen kann.

Auf diesen und weiteren Grundgedanken fußen Öffentliches Recht einerseits und Privatrecht andererseits. Die Arbeitsweise des Juristen baut darauf auf.

7 Vergleiche: Hegel, Rechtsphilosophie.

8 „Billigkeit“ im Sinne einer umfassenden Berücksichtigung sämtlicher Bedürfnisse. Dem Richter, der über die Billigkeit einer Ermessensentscheidung zu urteilen hat, kommt hier alttestamentarische Königsmacht zu, indem von ihm erwartet wird, allwissend und allweise zu sein.

9 Bspw. BGHZ 41, 271. 